\title{
Look beyond What You See - Lessons Learnt in Endocrinology
}

\author{
Milanka Wattegama ${ }^{1}$, Sajith Siyambalapitiya ${ }^{2}$, Noel Somasundaram ${ }^{3}$ \\ 1,2Diabetes and endocrine unit, North Colombo Teaching Hospital, Ragama \\ ${ }^{3}$ Diabetes and endocrine unit, National Hospital of Sri Lanka
}

Copyright: This is an open-access article distributed under the terms of the Creative Commons Attribution License, which permits unrestricted use, distribution, and reproduction in any medium, provided the original author and source are credited (CC BY 4.0)

A 36-year-old male presented with blurred vision of his left eye. The appearance of his fundus is shown in figure (1a). Four years later, he presented with acute right-sided loin pain and during this episode, he was also found to have incidental hypertension of 140/100 with tachycardia. The contrast enhanced channels around the lesions. In addition to these 2 lesions, there was a heterogeneous enhancing soft tissue mass of $15 \mathrm{~mm}$ in diameter in the right renal cortex (fig $1 \mathrm{~b}$ ). The magnetic resonance computerized tomography scan done during this admission, shown in figure $(1 \mathrm{~b})$, revealed a contrast enhancing soft tissue mass of $8.1 \times 4.7 \times 4.1 \mathrm{~cm}$ and $5 \times 3.5 \times 4.4 \mathrm{~cm}$ in the right and left suprarenal regions, respectively. There were multiple hypertrophied vascular

Imaging of the brain showed a contrast enhancing $0.4 \mathrm{~cm}$ lesion in the right cerebellum as seen in (fig 1c).

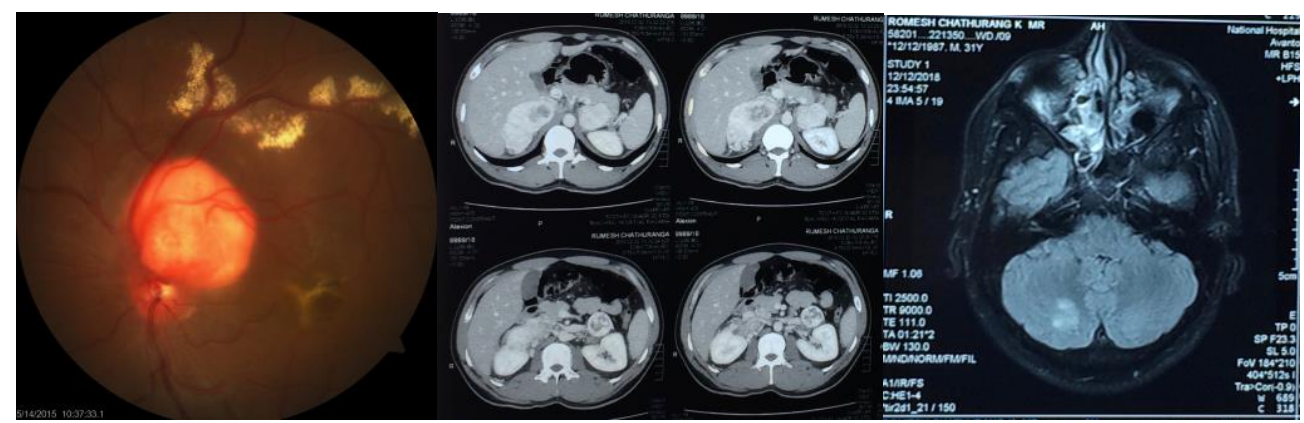

Figures 1a, 1b, 1c

What is the diagnosis?

\section{Von Hippel Lindau disease}

Von Hippel Lindau (VHL) is a rare autosomal dominant syndrome. Retinal and cerebellar haemangioblastomas, clear cell renal carcinomas, pheochromocytoms, pancreatic endocrine tumours and endolymphatic sac tumours are commonly seen among VHL patients. Retinal angiomas are the most common presenting feature of VHL and are frequently multiple and bilateral, usually present in mid-twenties. Dilated and tortuous vessels leading to and from the tumour are characteristic. Continuous growth of the tumour can lead to retinal hard exudates, retinal oedema and subsequently retinal detachments (2). CNS haemangiomas are seen in $40 \%$ of cases and usually arise in early thirties (2). These are usually found in the spinal column, cerebellum and the brain stem. Pheochromocytoma is found in 10 -
$20 \%$ of VHL patients. Mean age of presentation is 30 years. They can be multiple and bilateral. (4). Renal cell carcinomas are the commonly seen malignant neoplasms. The mean age of presentation is 39 years. Occasionally, advance tumours can present with flank pain as was seen in the indexed patient (3).

Deciding on the best management option for the bilateral pheochromocytomas was a challenging decision in this patient. Bilateral adrenalectomy invariably pushes the patient into lifelong steroid dependence and associated poor quality of life, with a lifelong risk of life threatening adrenal crisis. Cortical sparing adrenalectomy might be helpful in mitigating this problem. However, the probability of recurrence outweighs the benefits in these kinds of hereditary pheochromocytomas. This is an inherited disorder with multiple problems at different ages of life and this case underscores the importance of regular surveillance in VHL disease. 
1. Dollfus H, Massin P, Taupin P, Nemeth C, Amara S, Giraud S et al. Ocular manifestations in von HippelLindau disease: a clinical and molecular study. Investigative Ophthalmololgy and Visual science 2002; 43 : 3067-3074.

2. Maher ER, Yates JR, Harries R, Benjamin C, Harris R, Moore AT et al. Clinical-Features and NaturalHistory of von Hippel-Lindau Disease. Quarterly Journal of Medicine 1990; 77: 1151-1163.

3. Walther MM, Reiter R, Keiser HR, Choyke PL, Venzon D, Hurley K et al. Clinical and genetic characterization of pheochromocytoma in von Hippel-Lindau families: comparison with sporadic pheochromocytoma gives insight into natural history of pheochromocytoma. Journal of Urology 1999; 162 : 659-64. 